\title{
Effects of System Parameters and Controlled Torque on the Dynamics of Rigid-Flexible Robotic Manipulator
}

\author{
Sachindra Mahto \\ Mechanical Engineering Department, NERIST, Arunachal Pradesh-791109, India \\ E-mail:smh@nerist.ac.in \\ www.nerist.ac.in
}

\begin{abstract}
This work illustrates the effects of various system parameters on the dynamics of flexible link of revolute-jointed rigid-flexible manipulator. Flexible link is considered as a Euler-Bernoulli beam and finite element based on Langrange approach is employed for dynamic analysis. A comparative study is carried out for comparative dynamic response for the variation of system parameters and controlled torque excitation.
\end{abstract}

Keywords: Rigid-flexible revolute manipulator, Euler-Bernoulli beam, Shape optimization, Finite element method, Parametric study

\section{Introduction}

In the last few decades, dynamics and control of the flexible manipulators have received a considerable research attention. Flexible robotics systems have several advantages over the convention systems. However, due to lightweight of flexible systems, stiffness is low and there is a serious problem of vibration. Sometimes, to retain the advantages of the flexible system, some of the links are made flexible and some are rigid. Still the dynamic behavior improvement, optimal design and proper control strategy is the research interest for flexible manipulator.

Most of the researchers contributed their works in different ways of dynamic modeling and control aspects of flexible robotic manipulators. Sunada and Dubowsky [1] developed a lumped parameter FEM model for analyzing the complete behaviour of industrial robotic manipulator with complex-shape flexible links. Fakuda and Arakawa [2] studied the modeling and dynamic characteristics of two-link flexible robotic arms and controlled the vibration by taking into account the gravity, payload, and the coupled vibration between the first and second arm. Usoro et al. [3] developed finite element models to describe the deflection of a planar multi-link model. Ower and Vegte [4] used a Lagrangian approach to model the planar motion of a manipulator consisting of two flexible links and two rotary joints. Benati and Morro [5] developed a Lagrangian approach for the dynamics of chain with flexible links..

Bayo [6] used FEM to deal with multi-link flexible manipulator considering Timoshenko beam theory and including nonlinear coriolis and centrifugal effects for the elastic behavior. Jonker [7] presented a nonlinear finite element based formulation for analyzing the dynamic behavior of flexible manipulators. De Luca and Siciliano [8] presented closed-form equations of motion for planar flexible multi-link robot arm. Morris and Madani [9] studied the accurate modeling based on Lagrange-Euler formulation of a two link flexible manipulators. 
Dogan and Iftar [10] carried out the modeling and control of two-link robot manipulator whose first link is rigid and second link is flexible. Everett et al. [11] showed that it is possible to design a two-link flexible manipulator that has nearly position invariant first natural frequency with wide separation between the first two natural frequencies, to have its behaviour like rigid manipulator. Chen [12] developed a linearized dynamic model for multi-link planar flexible manipulator. Yang et al. [13] studied the tip trajectory tracking control for flexible multi-link manipulator using Lagrangian assumed mode method. Zhang et al. [14] derived a partial differential equation model for a flexible two-link manipulator using Hamilton's principle.

Some researchers presented their works on shape optimization of static/rotating beams. Karihaloo and Niordson [15] determined the optimum tapering of a cantilever beam carrying an end mass to maximize fundamental frequency. Wang [16] addressed optimum design of a single link manipulator to maximize its fundamental frequency. Yoo et al. [17] used the assumed mode method for dynamic modelling of rotating flexible manipulator for modal analysis and shape optimization to increase the fundamental frequency of the beam. Dixit et al. [18] presented a finite element model of single link robotic manipulator for revolute as well as prismatic joint. They used SQP for optimizing beam shapes under different optimization conditions.

From the above survey, it is observed that there is no much research contribution for the effects of system parameters in the dynamics of rigid-flexible robotic manipulator. In this paper, the dynamic behavior of robotic manipulator is presented for the variation of system parameters and improvement through control strategy.

\section{Modeling and Solution Technique}

The finite element formulation has been adopted here as described by Usoro et al. [3]. Fig. 1(a) shows rigid-flexible manipulator comprised of two links, two joints and tip load. The links are clamped on the joints. Shoulder joint (joint 1) is located at the origin of XOY represents the stationary co-ordinate frame and elbow joint (joint 2) is located at the origin of $\mathrm{X}_{1} \mathrm{O}_{1} \mathrm{Y}_{1}$ and $\mathrm{X}_{2} \mathrm{O}_{1} \mathrm{Y}_{2}$ which represent the moving co-ordinate frames. manipulator is considered slender. Flexible link is treated as a Euler-Bernoulli beam and gravity force is neglected.

Consider a point $P$ in the $i^{\text {th }}$ element on the manipulator at a distance ' $x$ ' from the elbow hub. The point $P$ attains the position $P^{\prime}$ with respect to inertial frame of reference (XOY) after having rigid body motion $\theta_{1}(t)$ and $\theta_{2}(t)$ of shoulder and elbow joint respectively and flexural deflection $w(x, t)$. Flexural deflection $w(x, t)$ of point $P$ is approximated in finite element technique using Hermitian shape functions as

$$
w(x, t)=N_{1} w_{2 i-1}+N_{2} w_{2 i}+N_{3} w_{2 i+1}+N_{4} w_{2 i+2}=\lfloor N\rfloor\{W\},
$$

where $\lfloor N\rfloor=\left\lfloor N_{1} N_{2} N_{3} N_{4}\right\rfloor$

and

$$
\{W\}^{T}=\left\lfloor\begin{array}{llll}
w_{2 i-1} & w_{2 i} & w_{2 i+1} & w_{2 i-2}
\end{array}\right\rfloor .
$$

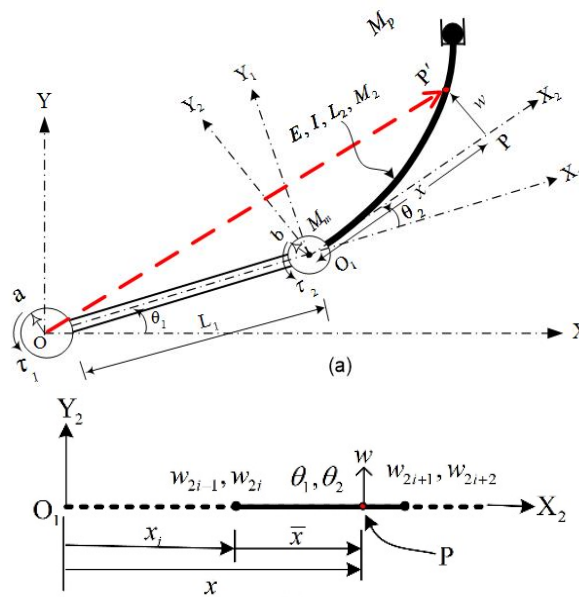

(b)

Fig. 1. (a) Configuration of rigid-flexible manipulator,

(b) Typical $i^{\text {th }}$ element with six dof

In FEM formulation, the manipulator is divided into finite number of elements with each element having six degrees of freedom. Detail of $i^{\text {th }}$ element of the link is shown in Fig. 1(b), where $\theta_{1}$ and $\theta_{2}$ are the hub rotation of shoulder and elbow joints respectively, $w_{2 i-1}, w_{2 i}$ and $w_{2 i+1}, w_{2 i+2}$ are the transverse deflection and slope at the first and second node of the element respectively. The position vector of $\mathrm{P}^{\prime}$ with respect to inertial system $\mathrm{XOY}$ is given by Eq. 2. For the prediction of approximate dynamic behaviour of the optimized beams, smaller excitation torque is considered for linearization of system modelling. Global position vector of the point $P^{\prime}$ under smaller angular and flexural displacement is given by 
$\boldsymbol{r}=\boldsymbol{o} \boldsymbol{p}^{\prime}=\left[\begin{array}{c}X \\ Y \\ 1\end{array}\right]=\left[\begin{array}{c}a+L_{1}+b+(i-1) h+\bar{x} \\ \left(a+L_{1}\right) \theta_{1}+((i-1) h+\bar{x}+b)\left(\theta_{1}+\theta_{2}\right)+\lfloor N\rfloor\{W\} \\ 1\end{array}\right]$,

In finite element method, variables are converted into nodal variables.

$$
\boldsymbol{r}=\boldsymbol{o} \boldsymbol{p}^{\prime}=f\left(\theta_{1}, \theta_{2}, w_{2 i-1}, w_{2 i}, w_{2 i+1}, w_{2 i+2}\right) .
$$

Let $\quad Z=\left\lfloor\begin{array}{llllll}\theta_{1} & \theta_{2} & w_{2 i-1} & w_{2 i} & w_{2 i+1} & w_{2 i+2}\end{array}\right\rfloor, \quad$ then absolute velocity of the point $P$ of the flexible link is obtained as

$$
\frac{\partial \boldsymbol{r}}{\partial t}=\left\lfloor\frac{\partial \boldsymbol{r}}{\partial Z}\right\rfloor \dot{Z}^{T}
$$

\subsection{Kinetic Energy Computation of the Link Element}

The Kinetic energy of the $i^{\text {th }}$ element of the link is given by

$$
K_{i}^{e}=\frac{1}{2} \int_{0}^{h} m_{2}\left[\frac{\partial \boldsymbol{r}^{T}}{\partial t} \cdot \frac{\partial \boldsymbol{r}}{\partial t}\right] \mathrm{d} \bar{x} .
$$

We have

$$
\frac{\partial \boldsymbol{r}^{T}}{\partial t} \cdot \frac{\partial \boldsymbol{r}}{\partial t}=\dot{Z}^{T}\left[\frac{\partial \boldsymbol{r}}{\partial Z}\right]^{T}\left[\frac{\partial \boldsymbol{r}}{\partial Z}\right] \dot{Z} .
$$

Substituting Eq. 6 in Eq. 5, we have

$$
K_{i}^{e}=\frac{1}{2} \dot{Z}^{T}\left[\int_{0}^{h} m_{2}\left[\frac{\partial \boldsymbol{r}}{\partial Z}\right]^{T} \cdot\left[\frac{\partial \boldsymbol{r}}{\partial Z}\right] \mathrm{d} \bar{x}\right] \dot{Z},
$$

and the elemental mass matrix is given by

$$
\begin{aligned}
M_{i}^{e} & =\int_{0}^{h} m_{2}\left[\frac{\partial \boldsymbol{r}}{\partial Z}\right]^{T} \cdot\left[\frac{\partial \boldsymbol{r}}{\partial Z}\right] \mathrm{d} \bar{x} \\
M_{i}^{e} & =\left[\begin{array}{llllll}
M_{11} & M_{12} & M_{13} & M_{14} & M_{15} & M_{16} \\
M_{21} & M_{22} & M_{23} & M_{24} & M_{25} & M_{26} \\
M_{31} & M_{32} & & & & \\
M_{41} & M_{42} & P_{i}(4 \mathrm{X} 4) & & \\
M_{51} & M_{52} & & & \\
M_{61} & M_{62} &
\end{array}\right] .
\end{aligned}
$$

All the constants of the above matrix in Eq. 8 are obtained by proper integration.

\subsection{Elastic Potential Energy of the Link Element}

The potential energy of the $i^{\text {th }}$ element of link due to elastic deformation is given by
$V_{i}^{e}=\frac{1}{2} \int_{0}^{h} E I\left[\frac{\partial^{2} w}{\partial x^{2}}\right]^{2} \mathrm{~d} \bar{x}=\{W\}^{T} \frac{1}{2} \int_{0}^{h} E I\left\lfloor N^{\prime \prime}\right\rfloor T\left\lfloor N^{\prime \prime}\right\rfloor \mathrm{d} \bar{x}\{W\}$.

Thus, elemental stiffness matrix is given by

$$
\begin{gathered}
K_{i}^{e}=E I \int_{0}^{h}\left\lfloorN ^ { \prime \prime } | ^ { T } \left\lfloor N^{\prime \prime} \mid \mathrm{d} \bar{x}\right.\right. \\
K_{i}^{e}=\frac{E I}{h^{3}}\left[\begin{array}{cccccc}
0 & 0 & 0 & 0 & 0 & 0 \\
0 & 0 & 0 & 0 & 0 & 0 \\
0 & 0 & 12 & 6 h & -12 & 6 h \\
0 & 0 & 6 h & 4 h^{2} & -6 h & 2 h^{2} \\
0 & 0 & -12 & -6 h & 12 & -6 h \\
0 & 0 & 6 h & 2 h^{2} & -6 h & 4 h^{2}
\end{array}\right] .
\end{gathered}
$$

\subsection{Lagrange's Equation of Motion in Discretized Form}

First link is rigid and posses kinetic energy only. However, being second link flexible it posses both kinetic and potential energy. The kinetic energy and the potential energy of the system are obtained by computing energy of rigid link and the energy of each element of the flexible link and then summing over all the elements. The global mass matrix and global stiffness matrix can be obtained as

$$
T=\frac{1}{2}[\dot{q}]^{T}[M][\dot{q}] \text { and } V=\frac{1}{2}[q]^{T}[K][q]
$$

respectively.

Here $\quad[q]=\left[\begin{array}{llllllll}\theta_{1} & \theta_{2} & w_{1} & w_{2} & \cdot & w_{2 n+1} & w_{2 n+2}\end{array}\right\rfloor$ is the global nodal vector. The Lagrangian of the system is given by $L=T-V$ and then Lagrange's equations of motion of this dynamic system may be written as

$$
\frac{\partial}{\partial t}\left[\frac{\partial L}{\partial \dot{\boldsymbol{q}}}\right]-\frac{\partial L}{\partial \boldsymbol{q}}=\boldsymbol{F}_{\boldsymbol{q}},
$$

where $\boldsymbol{F}_{q}$ is the generalized force vector. Due to modelling linearization, global mass and stiffness matrices are constant and equation of motion of undamped system is expressed as

$$
[M]\{\ddot{q}\}+[K]\{q\}=\{F\} .
$$

Global load vector $\{F\}$ and global nodal displacement vector $\{q\}$ for ' $n$ ' number of finite elements are given by $\{F\}=\left[\begin{array}{llllll}\tau_{1} & \tau_{2} & 0 & 0 & \ldots . .0 & 0\end{array}\right]^{\mathrm{T}}$. 


\section{Optimization Procedure}

To retain and optimize the advantage, upper limit of the optimized mass $\left(M^{*}\right)$ is constraint to the prescribed mass $(M)$. In the rigid-flexible robotic system, flexible link is considered for the shape optimization. $X=\left[\begin{array}{ll}d_{1} & d_{2}\end{array}\right.$ $\left.d_{\mathrm{n}}\right]^{\mathrm{T}}$ is the design vector with $d_{\mathrm{i}}$ indicating diameter of the $i^{\text {th }}$ finite element of link 2 .

Table 1. Different Optimization Problems

\begin{tabular}{c|c}
\hline Optimization Problem/Objective & Constraint \\
\hline $\begin{array}{c}\text { Maximization of fundamental beam } \\
\text { frequency }\end{array}$ & $\mathrm{M}^{*}-\mathrm{M} \leq 0$ \\
\hline \multicolumn{2}{c}{ Permissible Bound : $\mathrm{X}^{\mathrm{UB}}<\mathrm{X}<\mathrm{X}^{\mathrm{LB}}$} \\
\hline
\end{tabular}

Minimum and maximum diameter of the beam elements $(X)$ are denoted by $X^{L B}, X^{U B}$ respectively. The MATLAB function "fmincon" employing sequential quadratic programming (SQP) technique is used for constrained optimization of nonlinear function.

\section{Results and Discussion}

Modeling of this system is highly complex and nonlinear in nature. However, linearized model is preferred and considered in this work to predict its approximate dynamic behavior. Structural dimensions of this revolute-jointed rigid-flexible robotic manipulator for numerical experiments are taken as lengths $0.75 \mathrm{~m}$ and $0.75 \mathrm{~m}$, diameters $0.03 \mathrm{~m}$ and $0.01 \mathrm{~m}$, mass densities $2710 \mathrm{~kg} / \mathrm{m}^{3}$ and $2710 \mathrm{~kg} / \mathrm{m}^{3}$, hub radii $0.02 \mathrm{~m}$ and $0.01 \mathrm{~m}$, hub inertias $0.03 \mathrm{kgm}^{2}$ and $0.03 \mathrm{kgm}^{2}$ for first and second link respectively. Young's modulus $7.11 \times 10^{10} \mathrm{~N} / \mathrm{m}^{2}$ and mass of the motor $0.1 \mathrm{~kg}$ is considered.

Excitation torque $\tau_{=} \tau_{\mathrm{m}} \sin 2 \pi t \mathrm{Nm}$ is considered for both the joints for 2 seconds action. Torque amplitude $3.0 \mathrm{Nm}$ and $1.0 \mathrm{Nm}$ are considered for shoulder and elbow joints respectively. Static load of $1 \mathrm{~N}$ is considered at the tip of the manipulator for comparative static beam deflections. The ratio of payload $\left(\mathrm{M}_{\mathrm{p}}\right)$ to the mass of second link $\left(M_{2}\right)$ is denoted by $\mu_{2}$; ratio of motor mass $\left(M_{m}\right)$ to mass of the first link $\left(L_{l}\right)$ is denoted by $\mu_{1}$ and ratio of the second link length $\left(L_{2}\right)$ to first link length $\left(L_{l}\right)$ is denoted by $\left(L^{*}\right)$.

\subsection{Effects of Different Link Length}

As the second link is flexible, its length is varied with respect to the first link length. Effects of variation of link lengths in its natural frequencies, static tip deflection, hub angles, and dynamic response are plotted in Fig. 2 to Fig. 5. Natural frequencies of the flexible links decrease with increase of its length and vice versa. Static link deflection increases with increase of its length. Hub angles increase with decrease of its length and vice-versa. Residual vibration increases with increase of length of the flexible link.

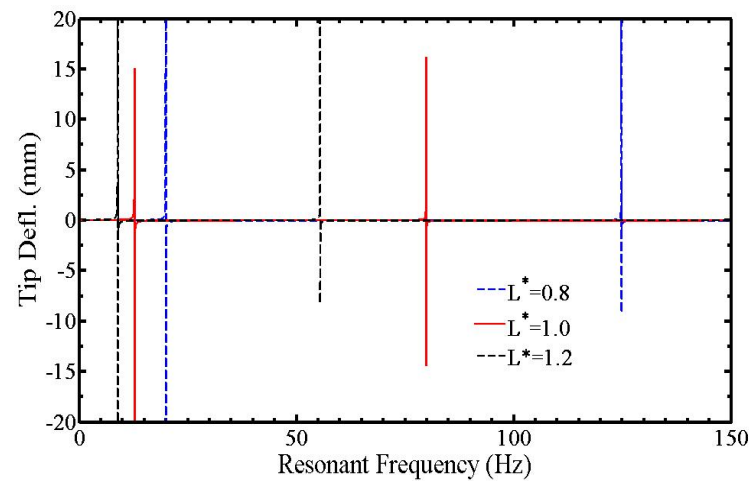

Fig. 2. Beam frequencies of flexible link for different link lengths

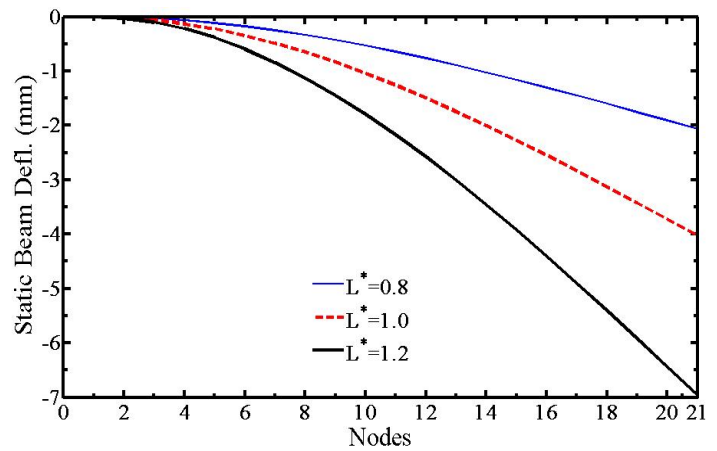

Fig. 3. Static deflection of flexible link due to $1 \mathrm{~N}$ force at tip for different link lengths
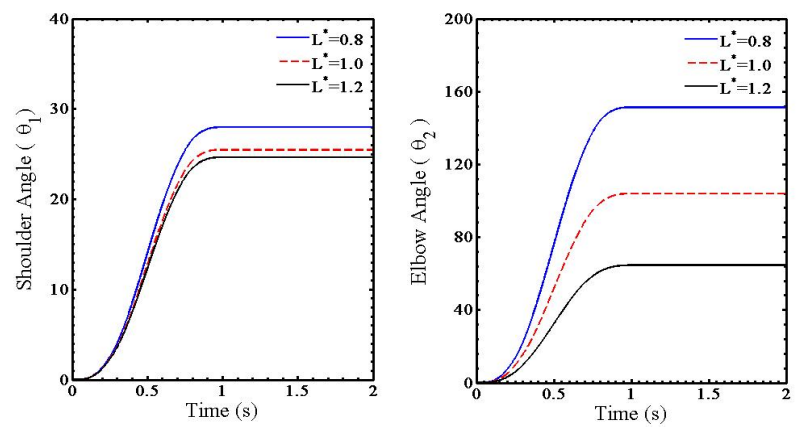

Fig. 4. Hub angles due to excitation torques for different link lengths 


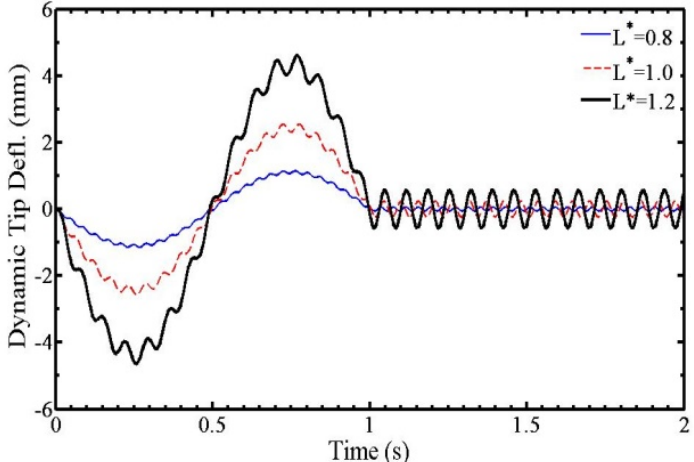

Fig. 5. Vibration residual of the tip of flexible link for different link lengths

\subsection{Effects of Different Payload, Motor Mass and Hub Inertia}

Robotic system means to take load and due to flexible links, there is always flexural vibration. Changes in the dynamics of the manipulator due to the change of payload, motor mass and hub inertia are plotted in Fig. 6 to Fig. 11. Hub angle decrease and residual vibration of flexible link tip increases with the increase of payload and vice-versa. By increasing the motor mass which is acting as a payload for link 1 has significant effect on shoulder joint angle for a given set of torques but less effect on elbow joint, however residual vibration increases. By increasing the hub-inertia of the shoulder joint, hub angles of shoulder joint decreases and elbow joint increase for a given set of applied torque and very less effect on the residual vibration of the flexible link tip.
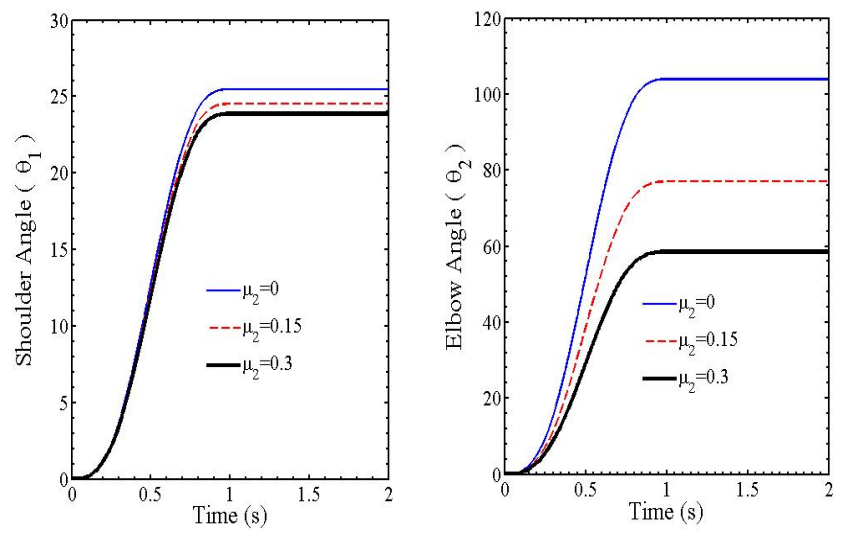

Fig. 6. Hub angles for different payloads for set of applied torques

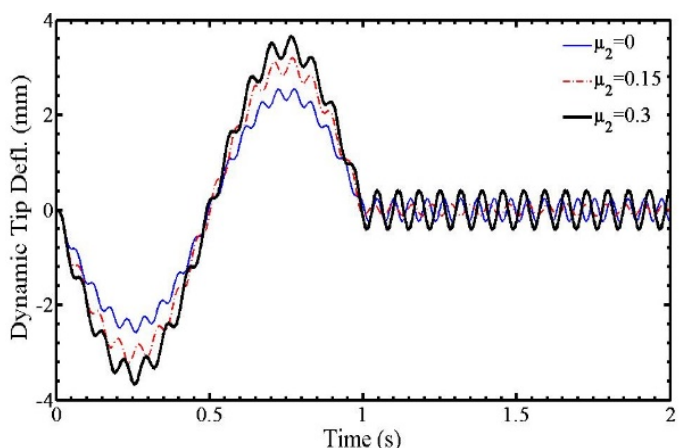

Fig. 7. Dynamic flexible tip deflection for different payloads
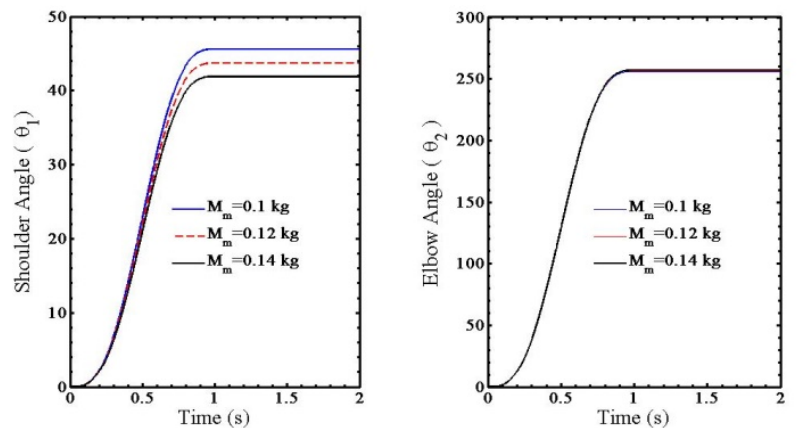

Fig. 8. Hub angles for different motor mass for set of applied Torques

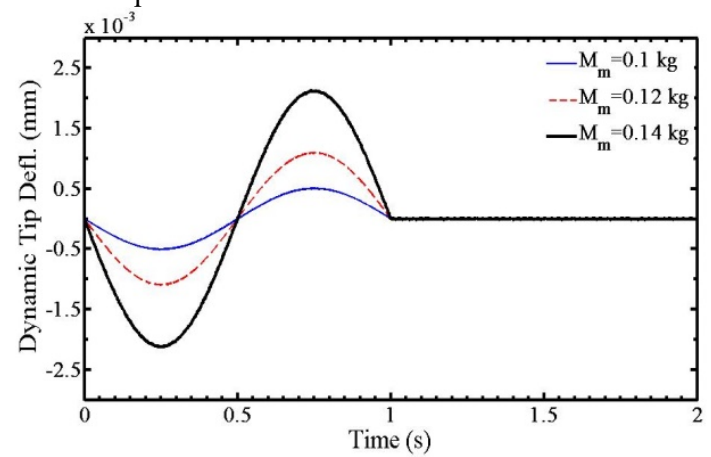

Fig. 9. Dynamic flexible tip deflection for different motor Mass
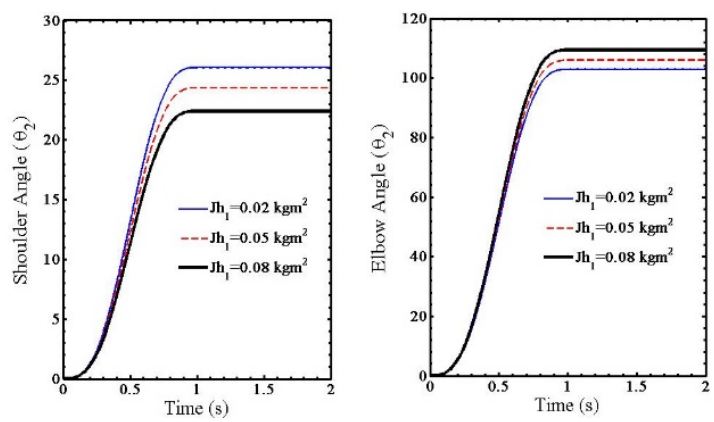

Fig. 10. Hub angles for different hub inertia for set of applied torques 


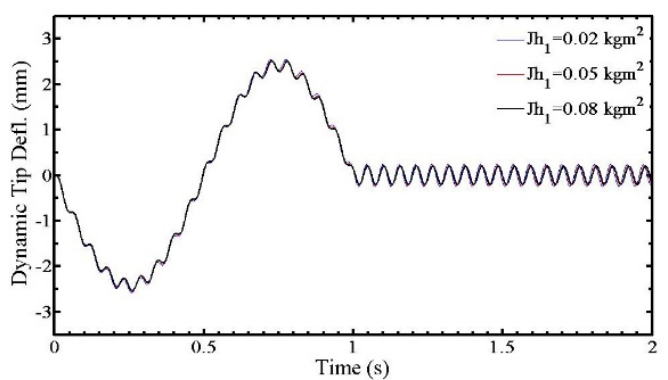

Fig. 11. Dynamic flexible tip deflection for different hub inertia

\subsection{Effects of Mass Distribution of Link}

Optimal design has a great importance in engineering applications. Shape optimization is done as per the optimization problem (Table 1) to increase the fundamental frequency and the optimized shape of the flexible link is shown in Fig. 12, where the mass of the link is re-distributed. It is observed that mass is concentrated more towards the root side in shape optimization. Static link deflection due to $1 \mathrm{~N}$ force at tip, natural frequencies of the optimized flexible link are plotted in Fig. 13 and Fig. 14 and its dynamics (hub angles and residual vibration) are plotted in Fig. 15 and Fig. 16 respectively for set of excitation torque. It is observed that parameters viz., static tip deflection, natural frequencies, hub angle and residual vibration are improved.

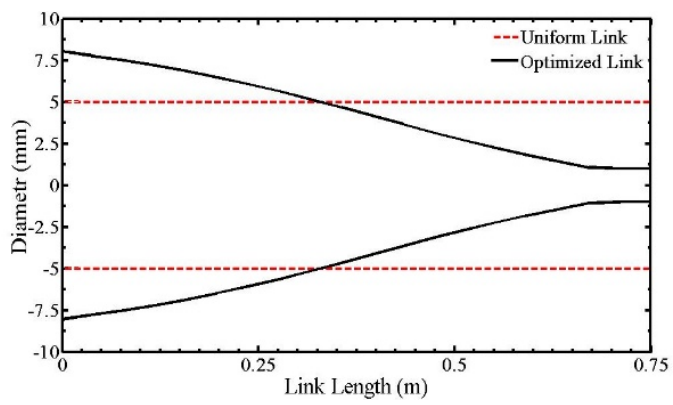

Fig. 12. Optimized shape of flexible link

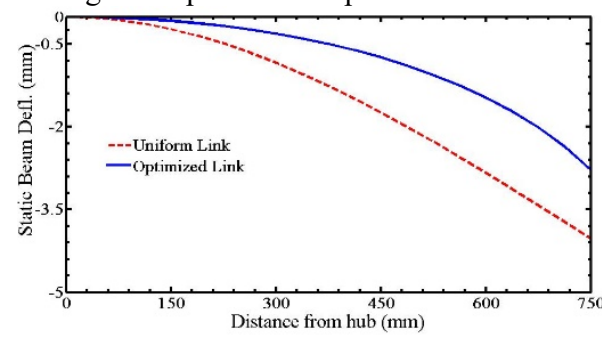

Fig. 13. Static beam deflection due to $1 \mathrm{~N}$ force at the tip of optimized link

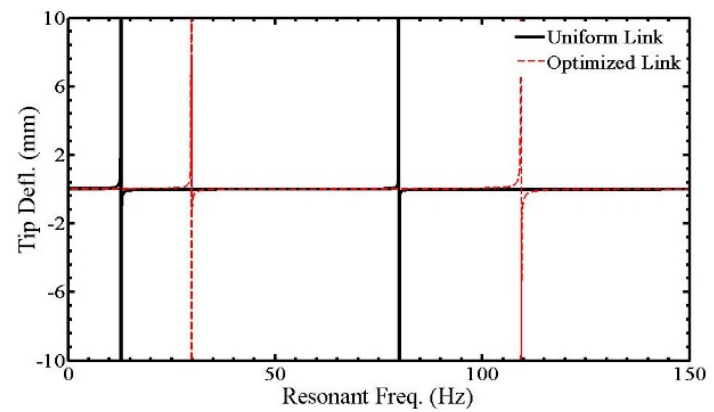

Fig. 14. Comparison of natural frequencies
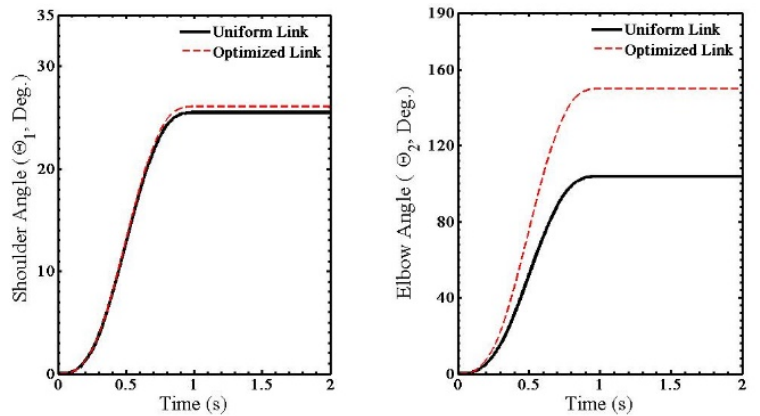

Fig. 15. Comparison of joint angles for set of applied torques

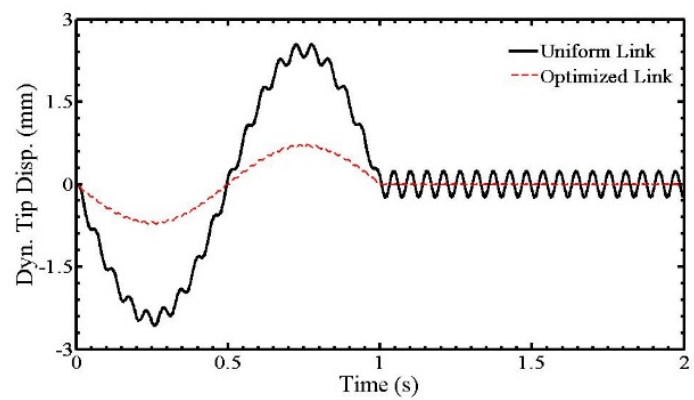

Fig. 16. Comparison of tip residuals for set of applied torques

\subsection{Effects of Controlled Torque}

Yigit [19] presented the position and derivative (PD) control torque for single link revolute-jointed flexible manipulator as given below

$$
\tau_{P D}=-K_{p}\left(\theta-\theta_{f}\right)-K_{v}(\dot{\theta}) .
$$

The feedback gains $K_{p}$ and $K_{v}$ depend upon the equivalent rigid system parameters and are expressed as

$$
K_{p}=\left(J_{h}+\frac{1}{3} m L^{3}+M_{p} L^{2}\right) f_{n}^{2},
$$

and

$$
K_{v}=2\left(J_{h}+\frac{1}{3} m L^{3}+M_{p} L^{2}\right) f_{n} .
$$


where ' $m$ ' is the mass per unit length, ' $L$ ' is the link length, ' $J_{h}^{\prime},{ }^{\prime} \theta_{f}^{\prime}$ ' is the final angular position, and ' $f_{n}$ ' is the fundamental frequency. PD controller is able to stabilize the system but vibration of the flexible beam can not be controlled. Ge et al. [20] extended the work of Yagit [19] and introduced energy-based robust (EBR) control law and added the nonlinear deflection feedback to improve the performance of the PD controller by adding the nonlinear control term as given by

$$
\tau_{E B R}=\tau_{P D}-K_{f} y(L, t) \int_{0}^{t} \dot{\theta}(\sigma) y(L, \sigma) \mathrm{d} \sigma,
$$

where $K_{f}$ is the gain constant of robust control, $\sigma$ is the dummy variable, $\dot{\theta}$ is the hub angular acceleration and $y$ is the deflection at the tips. Above control law is also applicable for multi-link robotic revolute jointed systems.

Elbow angle due to the controlled torque is plotted in Fig. 17 and residual vibration is shown in Fig. 18. It is observed that hub angle is achieved faster by controlled torque and tip vibration is lesser than the sinusoidal excitation.

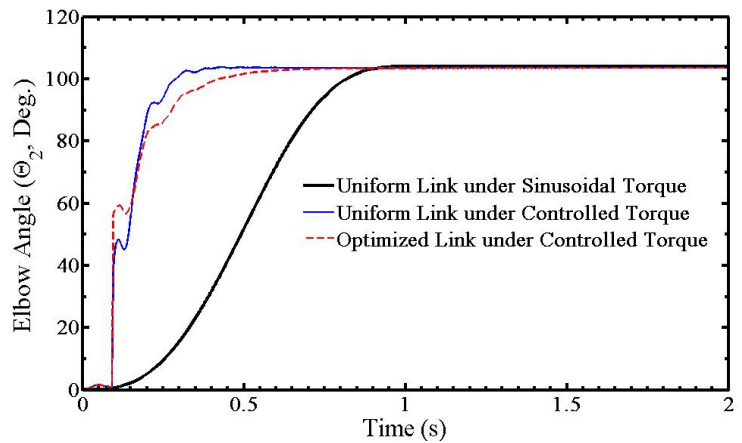

Fig. 17. Comparison of elbow angles for different excitation

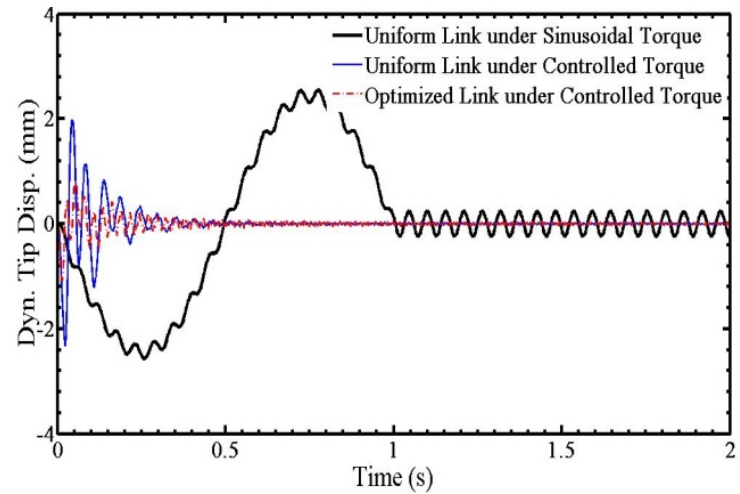

Fig. 18. Comparison of dynamic tip response for different excitation

\section{Conclusion}

In this work, finite element analysis of revolute-jointed rigid-flexible manipulator has been performed through linear modeling. Classical nonlinear optimization is used to solve the constrained shape optimization. From the numerical experiments, it is observed that there is a great role of system parameters (link lengths, payloads, hub-inertia, link shape) in its system dynamics and should be analyzed properly during the design. It is also observed that controlled torque improves the system dynamics further.

\section{References}

1. W. Sunada and S. Dubowsky, On the dynamic analysis and behaviour of industrial robotic manipulators with elastic members, ASME Journal of Mechanism, Transmissions and Automation in Design, 105(1) (1983), pp. 42-51.

2. T. Fukuda and A. Arakawa, Modeling and control characteristics for a two-degrees-of-freedom coupling system of flexible robotic arms, JSME, Series C, 30 (1987), pp. 1458-1464.

3. P.B. Usoro, R. Nadira and S.S. Mahil, S.S., A finite element/Lagrangian approach to modeling light weight flexible manipulators, ASME Journal of Dynamic System, Measurement and Control, 108 (1986), pp. 198-205.

4. J.C. Ower and J.V. Vegte, Classical control design for a flexible manipulator: modeling and control system design, IEEE Journal of Robotics and Automation, 3(5) (1987), pp. 485-489.

5. M. Benati and A. Morro, Dynamics of chain of flexible links, ASME J. of Dyn. Sys., Meas., and Control, 110 (1988), pp. 410-415.

6. E. Bayo, Timoshenko versus Bernoulli-Euler beam theories for inverse dynamics of flexible robots, International Journal of Robotics and Automation, 4(1) (1989), pp. 53-56.

7. B. Jonker, A finite element dynamic analysis of flexible manipulators, The International Journal of Robotics Research, 9(4) (1990), pp. 59-74.

8. A. DeLuca and B. Siciliano, Closed form dynamic model of planar multilink lightweight robots, IEEE Transactions on Systems, Man and Cybernetics SMC-21, 4 (1991), pp. 826-839. 
9. A.S. Morris and A. Madani, Static and dynamic modeling of a two-flexible-link robot manipulator, Robotica, 14(3) (1995), pp. 289-300.

10. A. Dogan and A. Iftar, Modeling and control of a two-link flexible robot manipulator, In Proceedings of the IEEE International Conference on Control Applications, 2 (1998), pp. 761-765.

11. L.J. Everett, T. Jennchen and M. Compere, Designing flexible manipulators with the lowest natural frequency nearly independent of position, IEEE Trans. on Robotics and Automation, 15(4) (1999), pp. 605-611.

12. W. Chen, Dynamic modeling of multi-link flexible robotic manipulators, Computers and Structures, 79(2) (2001), pp. 183-195.

13. T.W. Yang, W.L. Xu and S.K. Tso, Dynamic modeling based on real-time deflection measurement and compensation control for flexible multi-link manipulators, Dynamics and Control, 11(1) (2001), pp.524.

14. X. Zhang, W. Xu, S.S. Nair, and V.S. Chellabonia, PDE modeling and control of a flexible two-link manipulator,
IEEE Transactions on Control Systems Technology, 13(2) (2005), pp. 301-312.

15. B.L. Karihaloo and F.I. Niordson, Optimum design of vibrating cantilever. Journal of Optimization, Theory and Applications. 11(6) (1973), pp. 638-654.

16. F.Y. Wang, On the External Fundamental Frequencies of one Link Flexible Manipulators. The International Journal of Robotics Research, 13 (1994), pp. 162-170.

17. H.H. Yoo, J.E. Cho, and J. Chung, Modal analysis and shape optimization of rotating cantilever beams, Journal of Sound and Vibration, 290 (2006), pp. 223-241.

18. U.S. Dixit, R. Kumar, and S.K. Dwivedy, Shape optimization of flexible robotic manipulators, ASME Journal of Mechanical Design, 128 (2006), pp. 559-565.

19. A.S. Yigit, On the stability of PD control for a two-link rigid-flexible manipulator, J. of Dyn. Sys., Meas., and Control, 116 (1994), pp. 208-215.

20. S.S. Ge, T.H. Lee, G. Zhu, Energy-based robust controller design for multi-link flexible robots, Mechatronics, 6(7) (1996), pp. 779-798. 